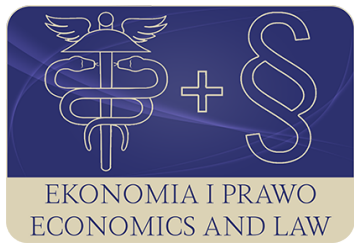

EKONOMIA I PRAWO. ECONOMICS AND LAW

Volume 20, Issue 3, September 2021

p-ISSN 1898-2255, e-ISSN 2392-1625

www.economicsandlaw.pl

EKONOMIA I PRAWO
ECONOMICS AND LAW

ORIGINAL ARTICLE

received 30.03.2021; revised 10.09.2021; accepted 30.09.2021

Citation: Elegeti, V. (2021). Rebalancing of exchange traded funds in stock market using option trading strategies. Ekonomia i Prawo. Economics and Law, 20(3), 513-527. https://doi.org/10.12775/EiP.2021.031.

\title{
Rebalancing of exchange traded funds in stock market using option trading strategies
}

\author{
VINAYKUMAR ELEGETI \\ Shri Jagdishprasad Jhabarmal Tibrewala University, Department of Commerce \& Management, \\ Sant dnyaneshwar ngr, Shri hari mandir marg, Bandra (E), Mumbai 400051, India \\ $\square$ elegetivinay@gmail.com \\ (D) orcid.org/0000-0002-7806-2784
}

\begin{abstract}
Motivation: The finance and academic industries are highly discussed in the stock market trading domain. The increase in economic globalization shows the connection among stock markets in different countries, which produces the effect of risk conduction in the market. Forecasting the direction of every day's stock market return is important and challenging. The growing complexity and dynamic features in stock markets are difficult in the financial industry. The inflexible trading method developed by financial practitioners utilized a larger amount of stock market features and is failed to achieve a satisfactory result in every condition of the market. Further, the existing data mining approaches are incomplete and inefficient.

Aim: To overcome the issues in stock and problem of existing methods, proposed option trading strategies for rebalancing Exchange Traded Fund (ETF) in the stock market. Rebalancing-ETF measure the volatility of the stock to track the error of model and rebalance the threshold quality to improve the trade. The proposed method increases the order of threshold quantity to rebalance the trade.

Results: The result showed that the minimum orders increases in rebalancing trade, which reduces the impact of price formations in market. The tracking error occurs when the larger quantity of threshold value reduces the quantity. Then, the markets are changed significantly when the Net Asset Values (NAV) of rebalancing ETF increases.
\end{abstract}

Keywords: exchange traded fund; financial industry; rebalancing; stock market; net asset values JEL: D00; E00; F00; G00; MOO 


\section{Introduction}

The indices of stock market prediction are most challenging for researchers and investors in forecasting the financial time series (Chen \& Hao, 2017). Generally, the financial times series caused by the facts of the stock market are unstable, changing the complex system dynamically, and nonlinear. Further, it is affected by various factors like government order economic policy, the variation in political condition, the future economy, investor's psychology, and so on (Das \& Padhy, 2017). Recently, the economic exchange among the country has become frequent which is near due to accelerating the financial unity partnered with the global economy (Cao et al., 2017). The finance market is a huge and dynamic field which is pretty much difficult to predict the model. This complex nature of financial markets has led people to occur statistical and mathematical approaches to make the stock market indices more evident and make the trading system more effective (Gudelek et al., 2017). However, certain gaps among the financial system and developed countries require to describe the stock market trading. The influences of India, China, and Brazil are the representative to emerge the country to grow their financial markets by describing linkage features such as long-term and downside risk spillovers with the developed stock markets (Li \& Wei, 2018).

The investors entered the stock markets to get a higher rate of returns with the income of a lower interest rate. But, the stock markets showed the volatility where the investors can gain the excess returns among the trading (Chang \& Lee, 2017). The trends of stock prediction are considered as the difficult task for financial time series. The convention of the statistical model technique was not suitable for stock trend forecasting due to the non-linearity and non-stationary of the stock markets

(Zhang et al., 2016). The market agents destroy the pattern of predictability and periodically raise the time series by having profit-seeking capacity (Pimenta et al., 2018). The profit-seeking characteristics made the time series of market non-stationary, harder for forecasting and temporary predictable model raised in financial series and was exploited (Huang \& Li, 2017). Instead of ending the field of a forecasting method in the stock market, the effective hypothesis of the stock market is associated with the motivation to establish innovative financial forecasting methods among the existing inflexible trading method that become unsuccessful (Wu et al., 2020). To overcome such an issue, the proposed method is a trading strategy to rebalancing the Exchange Traded Fund (ETF). The proposed method increases the order of threshold quantity to rebalancing the trade. This tracking error occurred which reduces the larger quantity of threshold value. Then, the markets are changed significantly when the Net Asset Values (NAV) of rebalancing ETF increases. This result shows minimum orders in rebalancing trade increases by reducing the impact of price formation in market. 
This paper is organized as follows, a survey of the existing methods is discussed in Section 2, the proposed rebalancing ETF method for the trading strategies of the stock market is explained in Section 3, experimental results are discussed in Section 4. The conclusion and future work of this research is given in Section 5.

\section{Literature review}

Golbabai \& Nikan (2020), developed a computational approach based on the moving least square technique for pricing the double barrier options in a time-fractional of Black Scholes. This developed method obtained the approximate solutions to the time fractions of the black Scholes model of $0<\leq 1$ order that governed the European option based on the Moving Least Squares (MLS) technique. Initially, the equation is discretized in time sense by using finite difference order which utilizes by the approximated MLS technique in the variable of space. The stability and convergence of the method were utilized to validate the process for pricing the double barrier option. This experimental result has shown that the developed method was accurate and efficient. But, it showed a higher range of error rate when more number of sample dataset was used to predict the stock market

Oscar et al. (2020), developed the Markov switching method with the Markov chain Monte Carlo inference technique for agriculture commodities trading. The developed approach was tested by using the active trading algorithm for the soybean and corn futures market. They assumed that investors live in two rules of the world such as lower and higher volatility periods. The trading algorithm assists the investors to forecast the probability in higher volatility rule at $\mathrm{t}+\mathrm{l}$. The Markov switching model is appropriate to generate the alpha from the passive investment strategy in the corn market and soybean trading. But, the developed method showed that the limitation to measure the higher volatility rules without knowing that higher volatility has been occurred due to bearish movements of prices.

Gambaro et al. (2020), developed a discrete-time model method with flexibility by changing the dynamics of driving for pricing the Asian option. The options are present everywhere in the market of finance by recasting the framework for pricing with the early practice and varying strikes of prices. The accurate read lesser bound that are evaluated the price of Europe and Asian options on stochastic volatility. This experimental result showed that the tree approach for Europe and Asian options in binomial approach was unconditional and convergent to continue the time equality. The developed method was monotonic, smooth, convergence, precise performance, and robust with the change in contract features. However, the developed method of computing time was more, when the step time was increased to fixed strikes and floating.

Dai et al. (2020), developed technical indicators that captured the latest information and the trends of the stock market. Then, the 2 step economic con- 
straints were developed with the better rule to forecast the larger variation that obtained the robust and stable prediction sample for performance. The 3 trading technical skills were used in relative strength indices, exponential moving average rules, and KDJ to sell or buy the signals based on the complex for simple mathematics function of present and past data of the stock market. This experimental result to return stock for a full sample period showed the significant prediction of capability from the technical indicator, However, from the weight of stocks, the risk disinclination factor affected the certainty equivalent return grains.

Yagi et al. (2020), developed a trading strategy of a leveraged ETF in the continuous double auction to predict stock market. In the developed approach the underlying assets of ETF were accessible for trading. The continuous trading session was used to identify the price of stocks. The price of selling orders in the order book is higher than the buying price and vice versa. The order of agents was matched immediately with the lower selling price in the book of order. The developed method increased the order quantity and reduced the overall threshold by rebalancing the quantity order that decreased the volatility in the market. However, the larger quantity of threshold value resulted in a higher period due to the error that occurred while tracking.

\section{Methods}

The proposed method is created with an agent of investors to manage the rebalancing assets based on artificial market methods. The simple agents and pricing technique is created that reproduced the statistical features and observed the types of longer-term price variations in the empirical analysis to find the relationship between 4 markets liquidity indicator by varying the parameter. Currently, in the proposed method, the two kinds of agents like a normal agents, general investors and rebalancing ETF agents that are explained in this section. The agents of investors are managed by the rebalancing assets to achieve the return rates in various times that are larger than rebalancing ETF agents. There are $n$ normal agents in general investors traded fund where, agent $j=1, \ldots, n$ and one rebalancing ETF in the markets.

By rebalancing the ETF risk assets that underplayed assets of rebalancing ETF are accessible for trading. The technique to identify the prices in proposed option trading strategies for rebalancing ETF method is continuous and double auctions or continuous trade sessions. There are buying and selling order costs in the order of book that is higher and lesser than the agents buying and selling order cost. The order of agents that immediately matched with the lesser selling orders or the maximum buying orders in the book of orders is called market orders. If the market orders are not present, then the orders will not match and will remain in the books of order. The remained orders in the books are cancelled at the time $t_{c}$ after placing the order, in which the order period is effective. The tickle size $\delta P$ is the minimum quantity to the prices. The agent 
possesses the indefinite assets due to the quantity of the indefinite agent's cash that reduces the selling. The proposed rebalancing ETF includes two processes like order process and the learning process.

Order Process: The two kinds of investor agents are described in the proposed method like normal agents and rebalancing agents. The normal agents have selected randomly and forward an order which has the quantity as one. Until the normal agents orders, the same agents will not order anything more than once. The rebalancing ETF agents will place the rebalancing order when it required where the normal agents place the order. When normal agents places the order when the rebalancing trade are not successful, time $t$ will be increased by 1 . Further, the process is forwarded to one step when new order was placed on the book and trades of normal agents are not occurred. The normal agent $j$ order of transaction price is calculated by using equation 1 . The rate of price changes are expected from agents $j$ during time $t$ and expected returns $r_{e j}^{t}$ is calculated by using equation 1 .

$$
r_{e j}^{t}=\frac{1}{w_{1 j}^{t}+w_{2 j}^{t}+u_{j}}\left(w_{1 j}^{t} r_{e 1, j}^{t}+w_{2 j}^{t} r_{e 2, j}^{t}+u_{j} \epsilon_{j}^{t}\right),
$$

where, $w_{1 j}^{t}$ is $i$-th term weight of agent, $j$ is the uniform distributions set related among 0 and $w_{i, \max }$ during simulation and it is assorted by utilizing the learning approach. The $u_{i}$ is the weight of 3 rd term which set the values based on uniform distribution between 0 and $u_{\max }$ in the simulation process, it has kept constant. There are three types of trading strategies that are included in the proposed method like technical process, fundamental process, and trading of noise is obtained among the agents. So, the trading weights are chosen independently by random variables.

The rate of prices change includes three types of trading process like technical process, fundamental process and noise process. In the proposed method, the trading process is implemented as a technical and fundamental strategy which is utilized normally to any markets at any time. Noise trading is also implemented to model the objectives of investors which is a desire for good strategies by trial and error approach. The risk assets which includes its fundamental costs are measured based on financial and economic factors that mean fundamental strategy. In contrast, the market prices are equal to the fundamental prices where the actual market prices are not equal to fundamental prices. The fundamental lists of the investors in fundamental strategy will identify the market prices which are connected with fundamental prices. When market prices are higher or lesser, the buying or selling of the stocks is connected to fundamental prices. The technical strategies focus on the pattern of trading signal, price movements, and many analytical charting tools that evaluated the strength and weakness of stocks. The trends of the present strategy are implemented in the proposed method to buy or sell the assets. When the trends of prices are 
higher for selling, the trade goes lower by expecting the movements of prices to continue. The investors in institution utilize the following trends of strategy in empirical estimation.

Initially, rate of prices change will normalize the effects of trading strategy. The lst term of parentheses in the right side of equation 1 describes the fundamental process of an agents who expect positive and negative returns during lower and higher market prices than the fundamental prices. The returns expected term of the fundamental strategies $r_{e l, j}^{t}$ for the agent $j$ at the time $t$ is given by equation 2 .

$$
r_{e l, j}^{t}=\ln \left(p_{f} / p^{t-1}\right),
$$

where, $p_{f}$ is fundamental prices that constant over time. The $p^{t}$ is the price of the market at time $t$, where the market prices are set according to recent prices if there is no trading occurred during the time. The prices of markets are set as the fundamental prices such as $p^{\circ}=p^{f}$.

The second terms are represented as the technical strategy that describes the expectation of agents as positive or negative returns. The term $r_{e 2, j}^{t}$ is the return of expected term in the technical strategy for the agent $j$ during the time $t$ which are expressed in equation 3.

$$
r_{e 2, j}^{t}=\ln \left(p^{t-1} / p^{\left(t-1-\tau_{j}\right)}\right)
$$

where, $\tau_{j}$ is basis of uniform distributions among 1 and $\tau_{\max }$ during the beginning of the simulation.

The third term is the rate of change of prices which describes the noise strategy in equation 4 . The $\epsilon_{j}^{t}$ is the random error that is normally distributed with the standard deviation $\sigma_{\epsilon}$ and mean zero. By using expected return $r_{e j}^{t}$ the expected prices $p_{e j}^{t}$ is estimated by using equation 4 .

$$
p_{e j}^{t}=p^{t-1} \exp \left(r_{e j}^{t}\right) \text {. }
$$

The order prices of $p_{0 j}^{t}$ is fixed based on the uniform distribution among the $p_{e j}^{t}-p_{d}$ and $p_{e j}^{t}+p_{d}$. Where, $p_{d}$ are constant that has choices among the selling and buying by identifying using the relative size of expected prices $p_{e j}^{t}$ and order price $p_{0 j}^{t}$;

- the agents place a buying order for the share if $>$;

- the agents place a selling order for the share if .

The rebalanced ETF agents place the orders when the overall rebalancing trades are required during the calculation which is explained in equation 5. The orders exceed the number of thresholds $V_{t h r}$. The $N A V^{t}$ and $L^{t}$ are the assets value and the actual leveraged ratio of rebalancing ETF during the time $t$ is calculated by using equations 5 and 6 . 


$$
\begin{aligned}
& N A V^{t}=P^{t-1} S^{t}+C^{t}, \\
& L^{t}=\frac{P^{t-1} S^{t}}{N A V^{t}}
\end{aligned}
$$

where, $S^{t}$ and $C^{t}$ is the overall assets for the cash that rebalancing ETF agents by possessed the time $t$.

The rebalancing ETF achieves the return rates with the targeted leveraged ratio of ETF $L$ at the times greater than the underlying assets. The rebalancing ETF agents trading begins when it satisfies the condition of $L$. The initial underlying asset is set as $S^{0}$ which is explained in equation 7.

$$
S^{0}=L C^{\text {init }} / P^{0},
$$

where the $C^{\text {init }}$ is initial cash after the rebalancing ETF at $C^{0}$ which is calculated by using equation 8 .

$$
C^{0}=C^{\text {init }}-P^{0} S^{0}=C^{\text {init }}-P^{0} L C^{\text {init }} / p^{0}=(1-L) C^{\text {init }} \text {. }
$$

The rebalanced ETF agents take the cash acquired by underlying assets when it is not satisfied with the targeted rebalanced ratio of ETF. The starting NAV of the rebalanced ETF agents $N A V^{0}$ is described as equation 9.

$$
N A V^{0}=P^{0} S^{0}+C^{0} .
$$

The total trades of rebalancing $V^{\mathrm{T}}$ is determined by using equation 10 .

$$
V^{t}=\frac{\left[\left\{(L-1) P^{t-1} S^{t}+L^{t} C^{t}\right\}\right]}{p^{t-1}} .
$$

When the value of $V^{t}$ exceeds the quantity of threshold value $V_{\text {thr }}$ he rebalancing trade of ETF agents is performed. The rebalancing ETF agents forward the selling orders when the sign of $V^{t}$ is positive and forward the buying order when the signs of $V^{t}$ is negative. The selling order and buying order is markets order as rebalancing ETF agents required to trade make $L^{t}$ equal to $L$.

Learning process: The proposed rebalancing ETF is implemented by the learning process of agents. The agents before ordering any stocks learn about it and represent the fundamental strategy of technical strategy by using the expected return $r_{e, j}^{t}$, where, $i=1,2$ at the time $t$ of the agent $j$ is determined by using equations 11 and 12 .

$$
\begin{aligned}
& r_{1, j}^{t}=\log P_{f} / P^{t}, \\
& r_{2, j}^{t}=r_{h, j}^{t} .
\end{aligned}
$$


The $r_{e i, j}^{t}$ learning is carried out by every agent before placing the order and returns the agent of the learning process $r_{l}^{t}$ as shown in an equation in 13 .

$$
r_{l}^{t}=\ln \left(\frac{P^{t-1}}{P^{t-1-t_{l}}}\right)
$$

where, $r_{l}^{t}$ returns to agents in the process of learning. The $p^{t}$ is the prices of the market at time $t$ and $t_{l}$ is the time during the learning process. The weight of $i$-th term to the agent $w_{i, j}^{t}$ is expressed in equation 14 .

$$
w_{i, j}^{t}+k_{l}\left|r_{l}^{t}\right| q_{j}^{t}\left(w_{i, \max }-w_{i, j}^{t}\right),
$$

where, $K_{l}$ is the constant and $r_{l}^{t}$ are the returns for agents in the process of learning, $q_{j}^{t}$ is the set as the basis of uniform distribution among 0 and 1 . The $r_{l}^{t}$ consists of different signs in which the $w_{i, j}^{t}$ value is updated as equation 15 .

$$
w_{i, j}^{t}-k_{l}\left|r_{l}^{t}\right| q_{j}^{t} w_{i, j}^{t} \text {. }
$$

The learning based process performance is done, the $w_{i, j}^{t}$ is updated with the smaller probability of $m$ based on the uniform distribution among 0 and $w_{i, \max }$ . To model the objectives of investors the good weights by trial and error are described. The learning method of rebalanced ETF agents are not modeled. The process of rebalanced ETF agents based on tracking errors.

\section{Results and discussion}

The rebalancing trade model influences underlying assets of market volatility which are possible to maintain and track the errors. This experiment is conducted to identify the rebalancing trade of reasonable methods by changing the threshold quantity of order. The analysis of the impact of rebalanced ETF agents NAV in underplayed to assets the markets by changing the initial cash of rebalancing ETF agents. The rebalancing ETF agents initial cost $C_{\text {init }}$ is defined as $C_{\text {init }}=1000000 \times C_{\text {mag }}$. The NAV is expressed as rebalancing ETF agents by varying the initial cash coefficient like $C_{\text {mag. }}$. The $C_{\text {mag }}$ is set as 10,20,30,40,50,60,70,80,90 and $100 C_{\text {mag }}$ is implemented to control the amount of $C_{\text {init }}$. If $C_{\text {init }}$ changes the effects of rebalancing ETF with various initial cash based on assets. The initial cash of rebalancing ETF in actual market are corresponding to $C_{\text {init }}$ not $C_{\text {mag }}$.

The $V_{t h r}$ is the order quantity of threshold to set based on five patterns like $1,2,3,4$, and 5. when the $C_{\text {mag }}$ is 10 which is greater than the values of $C_{\text {mag }}$ are shown in Table 1.

The $V_{\text {nor }}$ is introduced which is estimated by using the ratio of order quantity of threshold and initial cash coefficient $V_{\text {thr }} / C_{\text {mag }}$ for the discussion of pattern in an order quantity of threshold. The result of $V_{\text {nor }}$ indicates that orders quantity 
of threshold rises by increasing the coefficient of cash. Thus, the effect of the initial amount of cash required to consider the trades for rebalancing.

The $V_{\text {thr }}$ describes the minimum order of quantity to rebalance the trades. The rebalancing of trade is carried out when $V_{t h r}$ is lesser than the order quantity as per rebalancing trades. When the $V_{t h r}$ is changed the effects of order quantity per rebalancing the trades and overall rebalancing trades for the market of the underlying asset it is confirmed. When the $C_{\text {init }}$ is increased the quantity of order accordingly rebalancing trades also increased. In a proposed method, the $V_{\text {thr }}$ is utilized to order the quantity of threshold. The rebalancing trades increases when the $C_{\text {init }}$ is larger. So, the $V_{\text {nor }}$ and $V_{\text {thr }}$ is divided by $C_{\text {mag }}$ as the number of rebalancing trade will depend on the size of $C_{\text {init }}$. The $V_{t h r}$ is the minimum quantity of the order in the real market sets a lesser quantity of order to carry out rebalancing trade. By varying $V_{\text {nor }}$ the effects of order quantity in balancing the trades and rebalancing trade in market asset are changed.

Table 1 describes the $V_{\text {nor }}$ which set five values like $0.1,0.2,0.3,0.4$ and 0.5 . the $C_{\text {mag }}$ is set as $10,20,30,40,50,60,70,80,90$ and 100 . The simulation is carried out for every combination the result showed in an average of 100 simulations of run. Initially, the parameters value of proposed method are set as $n=1500$, $w_{1, \text { max }}=1, w_{1, \max }=5, u_{\text {max }}=2, \tau_{\text {max }}=20000, \sigma_{\epsilon}=0.05, k_{l}=5, m=0.01, p_{f}=10000$, $t_{\max }=1000000$ and $L=0.2$.

The statistical analysis of styled facts for the initial cash coefficients case is considered as 20 which is calculated for the price of returns for $\ln \left(\frac{p^{t}}{p^{t-1}}\right)$

in the intervals of 100 units of times. The autocorrelation coefficients and kurtosis of square shows various lags are positive which means the value of the run for every 5 patterns replicates the volatility clustering and fat tails. This approach replicates the longer terms of statistical features that are seen in financial markets. Similarly, the results are evaluated for different values of the initial cash coefficient due to the limitation of space. The statistical analysis of styled facts is shown in Table 2.

The results of overall rebalanced trade, quantity of total order for rebalancing, and order quantity per trade for rebalancing are shown in Tables 3 to 5 respectively. The number of rebalancing trade reduces the $V_{\text {nor }}$ and $C_{\text {mag }}$ that increases are in Table 3. The total quantity of order for rebalancing is decreased as the $V_{\text {nor }}$ increases but it increase as the Cmag increases that are shown in Table 4. The quantity of order per trade to rebalancing by increases the $V_{\text {nor }}$ and $C_{\text {mag }}$ which is shown in Table 5.

The results of overall quantity of order for rebalancing show that the order of quantity reduces the $V_{\text {nor }}$. Table 5 shows that rebalancing the quantity of the order is reduced by enhancing the value of the order quantity of the threshold.

Table 6 shows the volatility of markets and it increases when the $V_{\text {nor }}$ is lesser as the $C_{\text {mag }}$ more. But, the $C_{\text {mag }}$ increases much when the markets destroy by 
a pattern of predictability and periodically raises the time series by having profit-seeking capacity. This identification is obtained in rebalancing trades of rebalanced ETF which have larger NAVs with significant impacts on the markets to increase the volatility of a market. Table 7 . Shows the magnitude of changes from the previous market prices when the order quantity of rebalancing is equal to the order quantity of threshold.

\section{Conclusion}

The indices of stock market prediction are the most challenging problem in forecasting the financial time series for both researchers and investors. It is challenging and important to forecast the direction of every day's stock markets that are returned. The growing complexity and dynamic features in stock markets are difficult in the financial industry. In this research, an options trading strategy is proposed for rebalancing the ETF in the stock market. Initially, if the quantity order is increased it reduced the overall quantity of order for rebalancing and reduced the volatility of markets. Then, the NAV of rebalancing ETF is increased that resulted in the volatility of a market. The volatility of market significantly improved when the threshold quantity of order is smaller and has not changed when the threshold quantity of order is more. The proposed method increased the order of threshold quantity to the trade rebalancing. The tracking error occurred during the larger quantity which reduces the threshold value. Then, the markets are changed significantly when the NAV of rebalancing ETF increases. This result showed that when the minimum number of orders in the rebalancing trade increases by impacting the price formation that reduced in market.

\section{References}

Cao, G., Han, Y., Li, Q., \& Xu, W. (2017). Asymmetric MF-DCCA method based on risk conduction and its application in the Chinese and foreign stock markets. Physica A: Statistical Mechanics and its Applications, 468, 119-130. https://doi.org/10.1016/j.physa.2016.10.002.

Chang, Y.H., \& Lee, M.S. (2017). Incorporating Markov decision process on genetic algorithms to formulate trading strategies for stock markets. Applied Soft Computing, 52, 1143-1153. https://doi.org/10.1016/j.asoc.2016.09.016.

Chen, Y., \& Hao, Y. (2017). A feature weighted support vector machine and K-nearest neighbor algorithm for stock market indices prediction. Expert Systems with Applications, 80, 340-355. https://doi.org/10.1016/j. eswa.2017.02.044.

Dai, Z., Dong, X., Kang, J., \& Hong, L. (2020). Forecasting stock market returns: new technical indicators and two-step economic constraint method. The North American Journal of Economics and Finance, 53, 101216. https://doi. org/10.1016/j.najef.2020.101216. 
Das, S.P., \& Padhy, S. (2017). A new hybrid parametric and machine learning model with homogeneity hint for European-style index option pricing. Neural Computing and Applications, 28(12), 4061-4077. https://doi.org/10.1007/ s00521-016-2303-y.

Gambaro, A.M., Kyriakou, I., \& Fusai, G. (2020). General lattice methods for arithmetic Asian options. European Journal of Operational Research, 282(3), 1185-1199. https://doi.org/10.1016/j.ejor.2019.10.026.

Golbabai, A., \& Nikan, O. (2020). A computational method based on the moving least-squares approach for pricing double barrier options in a time-fractional Black-Scholes model. Computational Economics, 55(1), 119-141. https://doi.org/10.1007/s10614-019-09880-4.

Gudelek, M.U., Boluk, S.A., \& Ozbayoglu, A.M. (2017). A deep learning based stock trading model with 2-D CNN trend detection. In 2017 IEEE Symposium Series on Computational Intelligence, 1-8. https://doi.org/10.1109/ ssci.2017.8285188.

Huang, C.F., \& Li, H.C. (2017). An evolutionary method for financial forecasting in microscopic high-speed trading environment. Computational Intelligence and Neuroscience, 1-18. https://doi.org/10.1155/2017/9580815.

Li, X., \& Wei, Y. (2018). The dependence and risk spillover between crude oil market and China stock market: new evidence from a variational mode decomposition-based copula method. Energy Economics, 74, 565-581. https:// doi.org/10.1016/j.eneco.2018.07.011.

Oscar, V., Aguilasocho-Montoya, D., Álvarez-García, J., \& Simonetti, B. (2020). Using Markov-switching models with Markov chain Monte Carlo inference methods in agricultural commodities trading. Soft Computing, 24(18), 13823-13836. https://doi.org/10.1007/s00500-019-04629-5.

Pimenta, A., Nametala, C.A., Guimarães, F.G., \& Carrano, E.G. (2018). An automated investing method for stock market based on multiobjective genetic programming. Computational Economics, 52(1), 125-144. https://doi. org/10.1007/s10614-017-9665-9.

Wu, X., Chen, H., Wang, J., Troiano, L., Loia, V., \& Fujita, H. (2020). Adaptive stock trading strategies with deep reinforcement learning methods. Information Sciences, 538, 142-158. https://doi.org/10.1016/j.ins.2020.05.066.

Yagi, I., Maruyama, S., \& Mizuta, T. (2020). Trading strategies of a leveraged ETF in a continuous double auction market using an agent-based simulation. Complexity, 1-7. https://doi.org/10.1155/2020/3497689.

Zhang, X.D., Li, A., \& Pan, R. (2016). Stock trend prediction based on a new status box method and AdaBoost probabilistic support vector machine. Applied Soft Computing, 49, 385-398. https://doi.org/10.1016/j.asoc.2016.08.026. 


\section{Acknowledgements}

Author contributions: author has given an approval to the final version of the article.

Funding: this research was fully funded by the author's own sources. 


\section{Appendix}

Table 1.

The order quantity of the threshold $V_{t h r}$ for every condition

\begin{tabular}{|c|c|c|c|c|c|c|}
\hline \multirow{2}{*}{$C_{\text {mag }}$} & \multicolumn{6}{|c|}{$V_{\text {nor }}$} \\
\hline & & 0.1 & 0.2 & 0.3 & 0.4 & 0.5 \\
\hline & 10 & 1 & 2 & 3 & 4 & 5 \\
\hline & 20 & 2 & 4 & 6 & 8 & 10 \\
\hline & 30 & 3 & 6 & 9 & 12 & 15 \\
\hline & 40 & 4 & 8 & 12 & 16 & 20 \\
\hline & 50 & 5 & 10 & 15 & 20 & 25 \\
\hline & 60 & 6 & 12 & 18 & 24 & 30 \\
\hline & 70 & 7 & 14 & 21 & 28 & 35 \\
\hline & 80 & 8 & 16 & 24 & 32 & 40 \\
\hline & 90 & 9 & 18 & 27 & 36 & 45 \\
\hline & 100 & 10 & 20 & 30 & 40 & 50 \\
\hline
\end{tabular}

Source: Own preparation.

Table 2.

The stylized facts at $C_{\text {mag }}=20$

\begin{tabular}{|c|c|c|c|c|c|c|}
\hline \multirow{4}{*}{\begin{tabular}{l}
\multicolumn{1}{c}{ Kurtosis } \\
autocorrelation \\
coefficients for \\
the squared \\
returns
\end{tabular}} & \multicolumn{6}{|c|}{$V_{\text {nor }}$} \\
\hline & & 0.1 & 0.2 & 0.3 & 0.4 & 0.5 \\
\hline & & 9.93 & 8.56 & 6.15 & 4.45 & 4.13 \\
\hline & \multicolumn{6}{|c|}{ Lag } \\
\hline & 1 & 0.23 & 0.22 & 0.17 & 0.16 & 0.13 \\
\hline & 2 & 0.19 & 0.17 & 0.12 & 0.11 & 0.10 \\
\hline & 3 & 0.17 & 0.15 & 0.11 & 0.08 & 0.07 \\
\hline & 4 & 0.15 & 0.11 & 0.09 & 0.07 & 0.05 \\
\hline & 5 & 0.12 & 0.1 & 0.06 & 0.05 & 0.04 \\
\hline
\end{tabular}

Source: Own preparation. 
Table 3.

The overall of rebalancing trade

\begin{tabular}{|c|c|c|c|c|c|c|}
\hline \multirow[b]{2}{*}{$C_{\text {mag }}$} & \multicolumn{6}{|c|}{$V_{\text {nor }}$} \\
\hline & & 0.1 & 0.2 & 0.3 & 0.4 & 0.5 \\
\hline & 10 & 7.785 & 5.752 & 806 & 548 & 100 \\
\hline & 20 & 6.286 & 4.798 & 716 & 518 & 98 \\
\hline & 30 & 5.855 & 3.631 & 684 & 482 & 96 \\
\hline & 40 & - & 2.390 & 404 & 387 & 93 \\
\hline & 50 & - & 1.399 & 373 & 379 & 89 \\
\hline & 60 & - & - & 480 & 278 & 88 \\
\hline & 70 & - & - & 474 & 273 & 87 \\
\hline & 80 & - & - & 455 & 269 & 86 \\
\hline & 90 & - & - & 451 & 196 & 85 \\
\hline & 100 & - & - & 443 & 172 & 84 \\
\hline
\end{tabular}

Source: Own preparation.

Table 4.

The overall order quantity of rebalancing

\begin{tabular}{ccccccc}
\hline & \multicolumn{7}{c}{$V_{\text {nor }}$} \\
\hline$C_{\text {mag }}$ & \multicolumn{7}{c}{0.1} & 0.2 & 0.3 & 0.4 & 0.5 \\
\cline { 2 - 7 } & 10 & 12.851 & 5.595 & 2.337 & 1.028 & 508 \\
20 & 26.515 & 10.727 & 4.366 & 1.849 & 926 \\
& 30 & 41.780 & 14.557 & 6.176 & 2.569 & 1.318 \\
40 & - & 18.395 & 7.400 & 3.187 & 1.692 \\
50 & - & 21.401 & 8.517 & 3.806 & 2.058 \\
60 & - & - & 9.717 & 4.583 & 2.420 \\
70 & - & - & 10.859 & 5.151 & 2.844 \\
80 & - & - & 12.430 & 5.744 & 3.292 \\
90 & - & - & 13.782 & 6.721 & 3.731 \\
100 & - & - & 15.524 & 7.705 & 4.471 \\
\hline
\end{tabular}

Source: Own preparation. 
Table 5.

The quantity of per trade order for rebalancing

\begin{tabular}{ccccccc}
\hline & \multicolumn{7}{c}{$V_{\text {nor }}$} & & \\
\hline$C_{\text {mag }}$ & & 0.1 & 0.2 & 0.3 & 0.4 & 0.5 \\
\cline { 2 - 7 } & 10 & 2 & 4 & 6 & 8 & 10 \\
20 & 6 & 8 & 8 & 14 & 12 \\
30 & 12 & 14 & 12 & 18 & 16 \\
40 & - & 16 & 16 & 22 & 18 \\
50 & - & 18 & 18 & 24 & 28 \\
60 & - & - & 24 & 28 & 36 \\
70 & - & - & 26 & 32 & 42 \\
80 & - & - & 30 & 36 & 46 \\
90 & - & - & 36 & 42 & 48 \\
\hline
\end{tabular}

Source: Own preparation.

Table 6.

The underlying volatility of markets $\left(\times 10^{-3}\right)$

\begin{tabular}{ccccccc}
\hline & \multicolumn{7}{c}{$V_{\text {nor }}$} \\
\hline$C_{\text {mag }}$ & & 0.1 & 0.2 & 0.3 & 0.4 & 0.5 \\
\cline { 2 - 7 } & 10 & 1.45 & 0.95 & 0.81 & 0.79 & 0.78 \\
20 & 1.48 & 1.03 & 0.82 & 0.78 & 0.78 \\
30 & 3.27 & 1.07 & 0.81 & 0.79 & 0.78 \\
40 & - & 1.12 & 0.81 & 0.77 & 0.77 \\
50 & - & 1.16 & 0.8 & 0.76 & 0.77 \\
60 & - & - & 0.79 & 0.76 & 0.76 \\
70 & - & - & 0.78 & 0.74 & 0.75 \\
80 & - & - & 0.78 & 0.73 & 0.74 \\
90 & - & - & 0.78 & 0.73 & 0.74 \\
\hline
\end{tabular}

Source: Own preparation.

Table 7.

The changes in magnitude from previous price of market prices when the order quantity of rebalancing is equal to threshold

\begin{tabular}{lccccc}
\hline & \multicolumn{7}{c}{$V_{\text {nor }}$} \\
\hline price change & 0.1 & 0.2 & 0.3 & 0.4 & 0.5 \\
\cline { 2 - 6 } & 10 & 20 & 30 & 40 & 50 \\
\hline
\end{tabular}

Source: Own preparation. 
